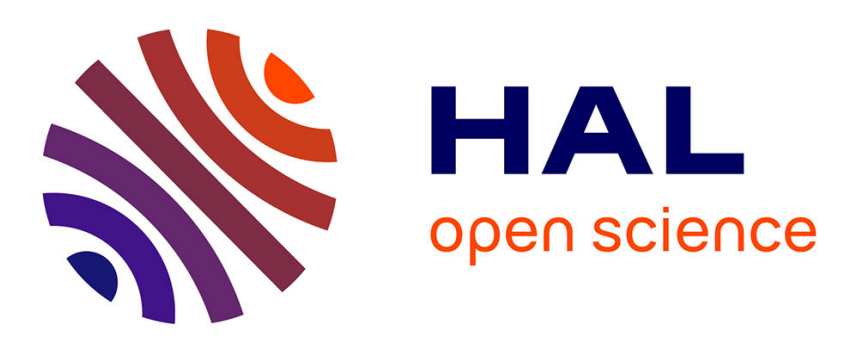

\title{
Is there a cluster of high theta-beta ratio patients in attention deficit hyperactivity disorder?
}

Aurore Bussalb, Sidney Collin, Quentin Barthélemy, David Ojeda, Stéphanie

Bioulac, Hilario Blasco-Fontecilla, Daniel Brandeis, Diane Purper Ouakil, Tomas Ros, Louis Mayaud

\section{To cite this version:}

Aurore Bussalb, Sidney Collin, Quentin Barthélemy, David Ojeda, Stéphanie Bioulac, et al.. Is there a cluster of high theta-beta ratio patients in attention deficit hyperactivity disorder?. Clinical Neurophysiology, 2019, 10.1016/j.clinph.2019.02.021 . hal-02149404

\section{HAL Id: hal-02149404 \\ https://hal.science/hal-02149404}

Submitted on 6 Jun 2019

HAL is a multi-disciplinary open access archive for the deposit and dissemination of scientific research documents, whether they are published or not. The documents may come from teaching and research institutions in France or abroad, or from public or private research centers.
L'archive ouverte pluridisciplinaire HAL, est destinée au dépôt et à la diffusion de documents scientifiques de niveau recherche, publiés ou non, émanant des établissements d'enseignement et de recherche français ou étrangers, des laboratoires publics ou privés. 


\title{
Is there a cluster of high theta-beta ratio patients in Attention Deficit Hyperactivity Disorder?
}

\author{
Aurore Bussalba,b, Sidney Collina, Quentin Barthélemy ${ }^{a}$, David Ojeda ${ }^{a}$, Stephanie \\ Bioulac $^{c, d, e}$, Hilario Blasco-Fontecilla ${ }^{f}$, Daniel Brandeis ${ }^{g, h, i, j}$, Diane Purper Ouakil ${ }^{k}$, Tomas \\ Ros', and Louis Mayauda
}

aMensia Technologies SA, 130 rue de Lourmel, 75015 Paris, France.

${ }^{b}$ Assistance Publique-Hôpitaux de Paris, Robert Debré Hospital, Child and Adolescent Psychiatry, Paris, France.

'Université de Bordeaux, Sommeil, Addiction et Neuropsychiatrie, USR 3413, F-33000 Bordeaux, France.

${ }^{\mathrm{d} C N R S}$, SANPSY, USR 3413, F-33000 Bordeaux, France.

eCHU Pellegrin, Clinique du Sommeil, F-33076, Bordeaux, France Universitaire

fDepartment of Psychiatry, Puerta de Hierro University Hospital-IDIPHIPSA, Autonoma University of Madrid, CIBERSAM, Madrid, Spain.

gDepartment of Child and Adolescent Psychiatry and Psychotherapy, Central Institute of Mental Health, Medical Faculty Mannheim/Heidelberg University, Mannheim, Germany. hDepartment of Child and Adolescent Psychiatry and Psychotherapy, Psychiatric Hospital, University of Zurich, Zurich, Switzerland.

'Neuroscience Center Zurich, University of Zurich and ETH Zurich, , Zurich, Switzerland. iZurich Center for integrative Human Physiology, University of Zurich, Zurich, Switzerland. ${ }^{k} \mathrm{CHU}$ Montpellier-Saint Eloi Hospital, University of Montpellier, Unit of Child and Adolescent Psychiatry (MPEA1), Montpellier, France.

'Department of Neuroscience, Geneva, Switzerland.

Corresponding Author: Aurore Bussalb aurore.bussalb@mensiatech.com

Running title: TBR Distribution Analysis in ADHD patients 


\section{Highlights}

- We offer significant insight into the standardization of theta-beta ratio (TBR) calculation;

- Our results suggest the presence of a distinct cluster of ADHD patients with an elevated TBR;

- This cluster makes up around 35\% of the ADHD population.

\section{Abstract}

Objective It has been suggested that there exists a subgroup of ADHD patients that have a high theta-beta ratio (TBR). The aim of this study was to analyze the distribution of TBR values in ADHD patients and validate the presence of a high-TBR cluster using objective metrics.

Methods The TBR was extracted from eyes-open resting state EEG recordings of 363 ADHD patients, aged $5-21$ years. The TBR distribution was estimated with three Bayesian Gaussian Mixture Models (BGMMs) with one, two, and three components, respectively. The pairwise comparison of BGMMs was carried out with deviance tests to identify the number of components that best represented the data.

Results The two-component BGMM modeled the TBR values significantly better than the one-component BGMM ( $p$-value $=0.005$ ). No significant difference was observed between the two-component and three-component BGMM ( $p$-value $=0.850)$.

Conclusion These results suggest that there exist indeed two TBR clusters within the ADHD population.

Significance This work offers a global framework to understanding values found in the literature and suggest guidelines on how to compute theta-beta ratio values. Moreover, using objective data-driven method we confirm the existence of a high theta-beta ratio cluster.

\section{Keywords}

Attention-Deficit/Hyperactivity Disorder; ADHD; EEG; Neurofeedback; clusters; thetabeta ratio 


\section{Introduction}

According to the National Institute of Mental Health, Attention-deficit/hyperactivity disorder (ADHD) is a "brain disorder marked by an ongoing pattern of inattention and/or hyperactivity-impulsivity that interferes with functioning or development." This disorder affects about $5 \%$ of people worldwide with varying degrees across the continents and countries (Polanczyk et al., 2007).

ADHD is heterogeneous in its symptoms as well as in its brain activity, which can be estimated using electroencephalographic (EEG) patterns (Barry et al., 2003; Liechti et al., 2013; Loo et al., 2013, 2018). It has long been reported that there are three subgroups of ADHD, categorized by the prevailing symptoms: predominantly hyperactive, predominantly inattentive, and combined. The latest Diagnostic and Statistical Manual of Mental Disorders (DSM-5) (The American Psychiatric Association, 2013) now regards ADHD subtypes as presentations because increasing evidence supports symptoms change across patient's lifespan (Centers for Disease Control and Prevention, 2017; Epstein et al., 2013). Diversity can also be observed in patients' EEG power patterns: the levels of energy that can be quantified for a given frequency, typically represented by delta $(0-4 \mathrm{~Hz})$, theta $(4-8 \mathrm{~Hz})$, alpha $(8-12 \mathrm{~Hz})$, beta $(12-32 \mathrm{~Hz})$, and gamma $(32-70 \mathrm{~Hz})$. Literature suggests there exists distinct clusters of ADHD patients separated using EEG power patterns, such as increased theta power and/or decreased beta power (Clarke et al, 2011; Loo et al., 2018). These power band differences can be quantified by calculating the ratio of the power in the theta band to the power in the beta band, or the theta-beta ratio (TBR) (Arns et al., 2013).

The TBR has been extensively researched as a diagnostic biomarker of ADHD. Some authors have suggested that increased TBR may help to confirm ADHD diagnoses (Arns et al., 2013). The U.S. Food and Drug Administration approved the use of TBR to aid in diagnosing ADHD (Neba Health, 2015; Saad et al., 2015; U.S. Food and Drug Administration, 2013). More recent studies failed to replicate a difference in the TBR values of ADHD patients, making its use as a diagnostic tool controversial (Zhang et al., 2017; Arns et al., 2013; Clarke et al., 2001). Instead, recent literature suggests that high 
TBR is a prognostic biomarker found in around 35\% of ADHD patients (Zhang et al., 2017; Arns et al., 2013; Clarke et al., 2001), which could be used to identify better responders to a given intervention.

A multimodal approach is recommended for the treatment of ADHD. This multimodal approach can combine different treatments according to the patients' needs and preferences: 1) medication (i.e. stimulants such as methylphenidate (MPH) or lisdexamfetamine; and/or non-stimulants: atomoxetine, guanfacine); 2) psychological treatment (i.e. cognitive behavior therapy, family therapy); and 3) psychoeducation. Pharmacological treatment is the most common treatment for ADHD in developed countries. However, this treatment has frequent side effects, including appetite suppression, growth delay or insomnia (Sousa et al., 2012). In addition, the long-term benefit of MPH medication is still a hot area of research (Storebo et al., 2015; Swanson et al., 2017) despite increasing evidence for positive long term medication effects from register studies using within-subject on-off control (Lichtenstein et al., 2012; Chang et al., 2017; Jangmo et al., 2019). For these reasons, and due to personal preferences, some parents and clinicians are reluctant to use this medication.

Drug-free treatment options for children with ADHD include behavioral therapy and dietary changes. Behavioral therapy uses a system of rewards to encourage children to control their ADHD (Evans et al., 2011; Sonuga-Barke et al., 2004). However, it has recently been shown that behavioral therapy (e.g parent management training) does not have a significant impact on ADHD symptoms but rather on oppositional-defiant symptoms and on familial relationships (Sonuga-Barke et al., 2013; Daley et al., 2014). Dietary changes include cutting out certain foods, i.e. dairy and/or sugar, or taking supplements, i.e. omega-3 fatty acids (Nigg and Holton, 2014). There is no strong evidence that dietary changes effectively treat ADHD symptoms (Nigg and Holton, 2014; Sonuga-Barke et al., 2013).

With the rise of neuroimaging, there is an increasingly popular view that psychiatric disorders are often characterized by disturbances at the molecular level, which in turn affects the functioning of interconnected brain networks (ICN) (Murphy and Basset, 2017), resulting in abnormal behavior. Pharmaceutical intervention addresses the lower level 
networks (micro/molecular/synaptic), while various forms of counseling address behavior (Antshel et al., 2011). In this context, Neurofeedback training (NFT) has long promised to be a drug-free alternative treatment for ADHD. NFT is a type of biofeedback that records EEG brain activity and represents it in the form of a digital visual and/or auditory signal that is used as feedback and can be modulated by the user (Marzbani et al., 2016). This allows the user to self-regulate the amplitude of specific frequency waves (Hernandez et al., 2016). The principle behind NFT is that it uses operant conditioning and skill learning to allow participants to learn to control their EEG and change their brain state to resemble that of a typically developing child (Van Doren et al., 2018). NFT has long demonstrated its efficacy on parental outcome, but it remains necessary to demonstrate the effectiveness from teachers' assessments which are presumed blind to the treatment (Cortese et al., 2016; Micoulaud-Franchi et al., 2014). The most common NFT protocols in the treatment of ADHD are TBR, sensorimotor rhythm (SMR), and slow cortical potentials (SCP) protocols (Arns et al., 2014; Mayer et al., 2016).

Arguably, one can increase the treatment effectiveness by allocating each patient to the training that would best address the patient's EEG phenotype. For instance, patients could be assigned to the lowering TBR protocol only if they have high TBR values. This method was proposed during the NEWROFEED trial (NCT02778360, Mensia Technologies, France, ClinicalTrials.gov), for which a choice on the TBR threshold had to be made. In a proposal for a double-blind randomized clinical trial of TBR neurofeedback for ADHD, the Collaborative Neurofeedback Group suggests using a TBR threshold of 5 (Collaborative Neurofeedback Group et al., 2013). However, this value was amended to 4.5 in the actual study (NCT02251743, Arnold, Ohio State University, ClinicalTrials.gov). A TBR threshold of 4.5 was also used in the NEWROFEED study (NCT02778360, Mensia Technologies, France, ClinicalTrials.gov). It is crucial that this threshold value be accurate (i.e. separate the data into homogeneous or meaningful clusters) because this value decides which NFT protocol the patient follows.

The arbitrariness of the TBR threshold values and the heterogeneity of methods employed certainly cast doubt on these decisions and calls for a sophisticated validation of the existence of a subgroup of ADHD patients with high TBRs, as well as the value of 
the threshold that separates these subgroups. Although previous literature points towards a cluster of ADHD patients with an increased TBR (Zhang et al., 2017, Clarke et al., 2011), no study has investigated the existence of clusters based solely on TBRs. The existence of two distinct ADHD subgroups, defined specifically by high or low TBR values needs to be validated. The threshold value that defines the cutoff point for the high TBR needs to be verified or revised. In addition to this, the large variability of TBR ranges reported will be investigated in the light of available technical implementation so as to make sense of the literature and guide future work.

The current study will examine the distribution of TBR values in children with ADHD and use cluster analysis to determine how many clusters exist in ADHD children based solely on TBR values. The threshold that separates these clusters will then be compared to previous thresholds used in literature to assign treatment protocols. We predict that there will be two clusters of ADHD patients based on TBR values.

\section{Methods}

The data used in this research study originated from three separate datasets: NEWROFEED (NCT02778360, Mensia Technologies, France, ClinicalTrials.gov), Child Mind Institute Multimodel Resource for Studying Information Processing in the Developing Brain (CMI-MIPDB) (Langer et al., 2017; Langer, 2017b), and Child Mind Institute Healthy Brain Network (CMI-HBN) (Alexander et al., 2017; Alexander, 2017b). For each database, written informed consent was obtained from all participants or their legal guardian. All the recordings were made in a controlled environment with eyes open at resting state during one minute under the supervision of a clinician or a researcher.

\subsection{Database description}

Table 1 provides a summary description of the three datasets as well as the additional inclusion criteria that were set to include patient in this study. The initial and final number of subjects eventually selected is also provided from each dataset. 
Table 1. Data information from all three separate datasets. The inclusion criteria for all datasets is specified, with the number of subjects that pass each criterion shown in parentheses. The total number of subjects included from each dataset is specified in the last row.

\begin{tabular}{|c|c|c|c|c|}
\hline Name & NEWROFEED & CMI-MIPDB & \multicolumn{2}{|c|}{ CMI-HBN } \\
\hline $\begin{array}{c}\text { Database } \\
\text { populations } \\
\text { description }\end{array}$ & $\begin{array}{l}\text { - 7-13 years old } \\
\text { - ADHD diagnosis } \\
\text { - Recorded with } \\
\text { Mensia Koala® } \\
\text { device (8 } \\
\text { electrodes) } \\
\text { international } 10-20 \\
\text { system }\end{array}$ & $\begin{array}{l}\text { - 6-44 years old } \\
\text { - With and without } \\
\text { clinical diagnoses } \\
\text { - 128-channel } \\
\text { EEG Geodesic } \\
\text { Hydrocel system }\end{array}$ & \multicolumn{2}{|c|}{$\begin{array}{l}\text { - 5-21 years old } \\
\text { - With and without clinical diagnoses } \\
\text { - 128-channel EEG Geodesic } \\
\text { Hydrocel system }\end{array}$} \\
\hline $\begin{array}{c}\text { Original } \\
\text { database } \\
\text { sample size }\end{array}$ & $\begin{array}{l}122 \text { (data available } \\
\text { in } 09 / 2017 \text { for } \\
\text { quality control } \\
\text { analysis before } \\
\text { study completion } \\
(12 / 2017) \text { ) }\end{array}$ & 126 & \multicolumn{2}{|l|}{881} \\
\hline $\begin{array}{c}\text { Additional } \\
\text { inclusion criteria } \\
\text { for this study }\end{array}$ & $\begin{array}{l}\text { 1. Age/diagnosis } \\
\text { info accessible } \\
\text { (122) } \\
\text { 2. ADHD } \\
\text { diagnosis (122) } \\
\text { 3. Accessible and } \\
\text { readable EEG } \\
\text { resting state } \\
\text { recordings with } 1 \\
\text { min eyes-open } \\
\text { (122) }\end{array}$ & $\begin{array}{l}\text { 1. Age/Diagnosis } \\
\text { info accessible } \\
\text { (126) } \\
\text { 2. ADHD } \\
\text { diagnosis (12) } \\
\text { 3. Accessible and } \\
\text { readable EEG } \\
\text { resting state } \\
\text { recordings with } 1 \\
\text { min eyes-open } \\
\text { (10) }\end{array}$ & $\begin{array}{l}\text { 1. Age/Diagnosis } \\
\text { info accessible } \\
\text { (447) } \\
\text { 2. ADHD } \\
\text { diagnosis (237) } \\
\text { 3. Accessible } \\
\text { and readable } \\
\text { EEG resting } \\
\text { state recordings } \\
\text { with } 1 \text { min eyes- } \\
\text { open (231) }\end{array}$ & $\begin{array}{l}\text { 1. Age/Diagnosis } \\
\text { info accessible } \\
(447) \\
\text { 2. Control group } \\
\text { - No diagnosis } \\
\text { given (76) } \\
\text { 3. Accessible and } \\
\text { readable EEG } \\
\text { resting state } \\
\text { recordings with } 1 \\
\text { min eyes-open } \\
(74)\end{array}$ \\
\hline $\begin{array}{l}\text { Final population } \\
\text { included in this } \\
\text { study }\end{array}$ & 122 & 10 & 231 & 74 \\
\hline
\end{tabular}

\subsubsection{NEWROFEED dataset}

At the time this work was conducted, the NEWROFEED study (NCT02778360, Mensia Technologies, France, ClinicalTrials.gov) was ongoing so the dataset at our disposal was an intermediate stage, not the final database, and consists of EEG data from 122 subjects diagnosed with ADHD according to the DSM-IV criteria (The American Psychiatric Association, 2013). The EEG was recorded using Mensia's Koala device, equipped with 8 individually-shielded silver-chloride electrodes placed according to the 
international 10-20 system located at Fpz, F3, Fz, F4, C3, Cz, C4, Pz. The EEG sampling rate was $512 \mathrm{~Hz}$. Impedances were kept below $40 \mathrm{kOhms}$ and level of electromagnetic contamination was kept below one third of the total signal energy. Additional information on the NEWROFEED study can be found at ClinicalTrials.gov (NCT02778360, Mensia Technologies, France, ClinicalTrials.gov). The inclusion criteria for this study are:

- Children or adolescents (male or female) aged 7-13 years;

- ADHD diagnosis positive with Kiddie-SADS (a semi-structured diagnostic interview based on the DSM-IV criteria) (Kaufman et al., 1997);

- ADHD RS IV > 6 for attention, with or without hyperactivity (Pappas, 2006).

Besides, children meeting at least one of these criteria were excluded:

- ADHD of the hyperactive/Impulsive subtype without inattention component;

- Severe and/or uncontrolled psychiatric disorder other than ADHD diagnosed with Kiddie-SADS such as autism, schizophrenia, severe generalized anxiety disorder, major depression or severe tics;

- Patient with comorbid disorder requiring psychoactive medication other than ADHD medication;

- IQ $<80$ using the 3-subtest form of the WASI or the WISC (Wechsler, 1999).

Only the first EEG assessment recorded for each patient was used for this study. The first assessment is recorded at the clinic under resting state condition (while the subject is not performing any task) during one minute with eyes-open and supervised by a clinician. The NEWROFEED clinical trial was conducted over 12 clinical centers in 5 European countries (France, Spain, Germany, Belgium, and Switzerland).

\subsubsection{Child Mind Institute Multimodel Resource for Studying Information Processing in the Developing Brain dataset}

The entire CMI-MIPDB dataset is composed of data from 126 participants, both typically developing and with clinical diagnoses (Langer et al., 2017; Langer 2017b). The participants were recruited from the Child Mind Medical Practice and the wider New York 
City area community. Each participant went through a 10-minute screening over the telephone or in-person, by a trained research assistant assessing participants' eligibility and safety to participate using:

- History of psychiatric illness, including past and present treatments, medications, and diagnoses;

- History of neurological disorders and/or epilepsy.

Patients were scheduled for EEG assessment if no contraindications were found (Langer et al., 2017).

Of all patients included in the CMI-MIPDB study, only those meeting the following criteria were selected for subsequent analysis:

a) patient is diagnosed with ADHD or ADD;

b) patient folder contains raw file with resting state EEG in '.raw' format;

c) age of patient is available.

Of the 126 patients available, only 10 (aged from 8 to 14 years old) met the inclusion criteria and were included in the study.

The high-density EEG data from the CMI dataset was recorded using a 128channel EEG Geodesic Hydrocel system at a sampling rate of $500 \mathrm{~Hz}$ and with a bandpass filter of 0.1 to $100 \mathrm{~Hz}$. The reference electrode was $\mathrm{Cz}$, the electrode located at the vertex of the head. Each participant's head circumference was measured so that the appropriately sized EEG net was used for recording. The electrode impedance was kept below $40 \mathrm{kOhms}$. Impedance was checked every 30 minutes as well as before each recording. Saline was added if necessary.

\subsubsection{Child Mind Institute Healthy Brain Network dataset}

The CMI-HBN dataset (Alexander et al., 2017; Alexander, 2017b) is composed of 881 patients, both typically developing and with clinical diagnoses. The participants were recruited using community-referred recruitment. The advertisements for the study specifically seek out families who are concerned about their child's psychiatric symptoms. 
Families were awarded $\$ 150$ for their participation. Participants were additionally offered consultation reports, feedback sessions, and referral information.

Only patients from the CMI-HBN study meeting the following criteria were included in this study:

a) patient is diagnosed with ADHD or ADD according to the KSADS-COMP (Kaufman et al., 1997);

b) patient folder contains raw file with resting state EEG in matlab format ('.mat');

c) age of patient is available.

Of the 881 patient files available, 231 patients (aged from 5 to 21 years old) met the inclusion criteria and were included in the current study.

The CMI-HBN dataset also contains healthy subjects (no diagnosis given), which will be used as prior for the Bayesian model described in Section 2.4. To be included in the analysis, subjects had no diagnosis and they had to meet b) and c) criteria cited above. 74 healthy subjects were finally selected (aged from 5 to 21 years old).

The EEG from the CMI datasets were recorded at the laboratory under the supervision of a researcher. Patients were under resting state condition with eyes open for one minute.

Data from different datasets went through different preprocessing primarily aimed at getting the data into compatible formats for comparison (electrode layout). All data went through the same processing to detect and remove artifacts as well as to extract TBR values.

\subsection{Pre-processing and standardization}

\subsubsection{NEWROFEED Dataset}

The only preprocessing that the NEWROFEED dataset required was temporal filtering. The signals were high-pass filtered at $0.5 \mathrm{~Hz}$ using a first order Butterworth filter to remove the DC component of the data, and band-stop filtered using a $47-53 \mathrm{~Hz}$ thirdorder Butterworth notch filter to remove artifact caused by electrical power lines. 


\subsubsection{CMI (HBN and MIPDB) Datasets}

The preprocessing for the CMI data included temporal filtering, removal and interpolation of noisy/disconnected channels, and spatial interpolation to downsample to the 8 international 10-20 electrodes used in the NEWROFEED dataset.

First, the resting state EEG signals were denoised and separated into eyes open and eyes closed files. Only the eyes open portion of the data was used for this analysis. The same temporal filtering that was applied to the NEWROFEED data, was also applied to the CMI data (except for the band-stop filtering: $57-63 \mathrm{~Hz}$ was applied here).

It is common on high-spatial resolution EEG that one of more channels occasionally disconnects resulting in both large amplitude artefact and zero lines. A special strategy was implemented to reliably detect and interpolate these disconnected channels. The variance of each EEG channel was calculated over ten-second-sliding windows. This variance was converted into two distinct z-scores using: (1) the instantaneous distribution of variances for all other 127 channels (spatial z-score) as well as (2) to the cumulative historical distribution of variances for the same channel (temporal $z$-score). If the temporal z-score was below -5 or above 5 , the signal was detected as an artifact and was interpolated from surrounding channels. If the spatial z-score was above 2 or less than -2 , the signal either had excess variance (meaning it was noisy) or insufficient variance (meaning the electrode was probably disconnected and not recording anything), and was detected as an artifact and interpolated.

The data was re-referenced to the left mastoid (EGI-128 electrode 57) electrode to match the referencing on the NEWROFEED dataset. The EEG signals were then spatially reconstructed from the 128 electrodes in the EGl electrode space to eight electrodes in the international 10-20 electrode space to match the other datasets: Fpz, F3, Fz, F4, C3, Cz, C4, Pz.

In order to interpolate from the $128 \mathrm{EGl}$ electrodes to the 8 standard $10-20$ electrodes, the coordinates of the $128 \mathrm{EGl}$ electrodes needed to be transformed to the standard 10-20 electrode space. The coordinates were scaled by projecting the values 
onto the unit sphere. The axes were rotated to verify the electrode coordinates matched. We assume these scaled coordinates are in the standard 10-20 electrode space.

The EEG was recorded at the laboratory under the supervision of a researcher. Patients were under resting state condition with eyes open for one minute.

\subsection{TBR extraction}

Once all the EEG data was preprocessed and the eyes open portion was isolated, Riemannian geometry was used to discard artifactual segments in the signals. Then, once the rejection of artifactual data is complete, the individualized alpha peak frequency (iAPF) was extracted for the computation of individualized frequency bands, and the TBR was calculated as described below. The method presented in this paper to calculate and compare TBR values on eyes open introduces steps to make the estimates of the TBR index more reliable.

First, artifactual data is removed from the original time series using the Riemannian Potato Field (RPF) (Barthélemy et al., 2019). EEG recordings were epoched (2 seconds length, overlapped every 0.125 second) and the covariance matrix of each epoch is computed for a subset of channels. Then, the RPF rejects epochs whose covariance matrices lie out of a region of acceptability defined thanks to a reference of clean EEG.

Second, the iAPF was calculated by finding the frequency at which the peak power was observed within the alpha band, defined as $7-13 \mathrm{~Hz}$. If no peak value is found, Klimesch's iAPF estimate, calculated from the subject's age, is used (Klimesch, 1999). The IAPF is calculated from the EEG measured on the Pz electrode from the eyes open resting state EEG recording.

Third, the frequency bands were personalized for each subject using the IAPF and the following definitions: theta $=[\mathrm{iAPF}-5 \mathrm{~Hz}, \mathrm{iAPF}-1 \mathrm{~Hz}]$, beta $=[\mathrm{iAPF}+3 \mathrm{~Hz}, \mathrm{iAPF}+12 \mathrm{~Hz}]$. This personalization step was meant to adjust for young and fast developing brains so as to not consider some of the lower alpha components as part of the theta band; arguably this method will provide with different results than fixed frequency band analysis (Arns et al., 2008, Vollebregt et al., 2015). The efficiency of the use of individualized bands have 
been the subject of several studies (Kaiser et al., 2001; Bazanova and Aftanas, 2006, 2008, Escolano et al., 2014), which shown that using this personalization could improve the efficiency of TBR NFT in ADHD.

The TBR was then calculated on eyes open: it is defined as the ratio of power in the theta frequency band to power in the beta frequency band. A moving average signal power $\bar{P}$ was estimated using Welch's method (Welch, 1967) on 32 epochs, with an epoch duration of 2 seconds, overlapped every $1 / 16$ second. This method for calculating power is specified below:

$$
\begin{gathered}
P_{c, b}=\left\|x_{c, b}\right\|_{F}^{2},(1) \\
\bar{P}_{c, b}=\frac{1}{I} \sum_{i=1}^{I}\left(P_{c, b}\right)_{i},(2)
\end{gathered}
$$

where $x_{c, b}$ denotes an epoch of EEG signal on channel $c$, band-pass filtered on the $b$ frequency band, $\|\cdot\|_{F}$ is the Frobenius norm, and I the number of epochs. The power in the theta and beta bands were calculated respectively, using the above equations, and divided to get their ratio TBR $=\bar{P}_{c, \theta} / \bar{P}_{c, \beta}$ giving an instantaneous ratio of power values that represents a useful real-time implementation of the TBR.

The median was used as a representative statistic of the whole recording and computed for $\mathrm{Fz}$ and $\mathrm{Cz}$. The max over these two electrodes was used. Finally, the TBR was normalized for the following analysis into a square-root normalized TBR (srnTBR) as:

$$
\operatorname{srnTBR}=\sqrt{\max \left(\bar{P}_{F z, \theta} / \bar{P}_{F z, \beta} ; \bar{P}_{C z, \theta} / \bar{P}_{C z, \beta}\right)} .
$$

\subsection{Modeling the TBR distribution}

The srnTBR distribution was plotted and fit to a Bayesian Gaussian mixture model (BGMM) (Attias, 2000; Blei et al., 2006). A Gaussian Mixture Model (GMM) is a probabilistic model made up of a finite number of Gaussian distributions; the model assumes that all the data points originate from a mixture of a finite number of Gaussian distributions with unknown parameters. A BGMM uses given priors on data distribution to 
assign posterior probabilities to each Gaussian mixture distribution defined by parameters. A large database of TBRs of healthy patients (no diagnosis given) from the CMI-HBN dataset was available, so it was used to estimate the priors for the BGMM. When data is fit to a BGMM, a Gaussian model made up of a preset number of Gaussian curves is created to model the data. The srnTBR values were fit to a BGMM and an increasing number of components.

The choice of a parsimonious modelling technique was made to validate the need of a second TBR cluster to explain observed distributions.

\subsection{Statistical analysis}

The significance of difference between each model was tested using a $\chi^{2}$ test on the model deviance (James et al., 2013). Deviance is a goodness-of-fit statistic for a statistical model, it is defined as:

$$
D=-2\left(\log \left(L\left(\beta_{0}\right)\right)-\log \left(L\left(\beta_{\alpha}\right)\right)\right),(4)
$$

where $\log \left(L\left(\beta_{0}\right)\right)$ is the log likelihood of the null model and $\log \left(L\left(\beta_{\alpha}\right)\right)$ is the $\log$ likelihood of the alternate model. Multiplying by -2 is a necessary step to convert the log likelihood into a chi-square distribution, which allows for the use of a $\chi^{2}$ test to test for statistical significance. An alpha value of .01 was used as the significance level.

\subsection{Identification of the optimal threshold for discrimination}

Using the BGMM offering the best model fit, the theoretical distribution of each component was used to infer to the threshold value offering the best discrimination between the two clusters. A receiver operating characteristic $(R O C)$ curve was also created from the two BGMM components to calculate the false positive rate (FPR) and true positive rate (TPR).

Results were compared to a nonparametric approach towards threshold identification using Ward's method of hierarchical clustering. Ward's clustering method is 
a type of agglomerative clustering that merges clusters based on minimizing intra-cluster variability (Ward, 1963). The data was clustered into two components and the threshold value separating the two clusters was obtained. This threshold was compared to the threshold obtained using the BGMMs.

Finally, we also compared our threshold to other thresholds from previous literature. The threshold of 5 and 4.5 are plotted on the ROC curve because the Collaborative Neurofeedback Group first suggests using a TBR threshold of 5 (Collaborative Neurofeedback Group et al., 2013), although in their actual clinical trial they used a threshold of 4.5 (NCT02251743, Arnold, Ohio State University, ClinicalTrials.gov) as well as the NEWROFEED study (NCT02778360, Mensia Technologies, France, ClinicalTrials.gov). The threshold that separates the largest 35\% of the TBR values was plotted as it is the suggestion from Zhang et al.'s recent study (Zhang et al., 2017) based on the results of Clarke et al.'s (Clarke et al., 2011). The 10\% FPR threshold was displayed as it would constitute an interesting prognosis strategy. In essence, you only want to treat the patients who belong to the high-TBR subgroup, with the down-TBR protocol.

All processing of EEG data was implemented under the NeuroRT software platform ( $\mathrm{v} 3$, Mensia Technologies, Paris, France) and statistical analysis was performed with Scikit Learn Python (v0.18.1, Pedregosa et al., 2011).

\section{Results}

\subsection{Population}

Patient demographics are detailed in Table 2 and the control demographics are detailed in Table 3. 
Table 2. Demographics of ADHD patients, separated by dataset and combined. $\mathrm{F}=$ Female; $\mathrm{M}=$ male; $\mathrm{N}$ $=$ number of patients. Standard deviation for mean age is shown in parentheses.

\begin{tabular}{|c|l|l|l|l|}
\hline & \multicolumn{1}{|c|}{ N } & \multicolumn{1}{|c|}{ Gender } & \multicolumn{1}{c|}{$\begin{array}{c}\text { Mean age } \\
\text { (years) }\end{array}$} & \multicolumn{1}{c|}{$\begin{array}{c}\text { Age range } \\
\text { (years) }\end{array}$} \\
\hline CMI-MIPDB & 10 & $3 \mathrm{~F} ; 7 \mathrm{M}$ & $11.30(2.15)$ & $8-14$ \\
\hline CMI-HBN & 231 & $57 \mathrm{~F} ; 180 \mathrm{M}$ & $10.04(3.41)$ & $5-21$ \\
\hline NEWROFEED & 122 & $24 \mathrm{~F} ; 98 \mathrm{M}$ & $9.66(1.85)$ & $7-13$ \\
\hline $\begin{array}{c}\text { All Datasets } \\
\text { Combined }\end{array}$ & 369 & $60 \mathrm{~F} ; 187 \mathrm{M}$ & $10.09(3.36)$ & $5-21$ \\
\hline
\end{tabular}

Table 3. Demographics of healthy controls (no diagnosis given). $\mathrm{F}=$ Female; $\mathrm{M}=$ Male; $\mathrm{N}=$ number of patients. Standard deviation for mean age is shown in parentheses.

\begin{tabular}{|l|l|l|l|l|}
\hline & \multicolumn{1}{|c|}{$\mathbf{N}$} & \multicolumn{1}{|c|}{ Gender } & \multicolumn{1}{c|}{$\begin{array}{c}\text { Mean age } \\
\text { (years) }\end{array}$} & $\begin{array}{c}\text { Age range } \\
\text { (years) }\end{array}$ \\
\hline $\begin{array}{l}\text { Healthy } \\
\text { Controls }\end{array}$ & 76 & $38 \mathrm{~F} ; 38 \mathrm{M}$ & $10.19(3.49)$ & $5-21$ \\
\hline
\end{tabular}

\subsection{TBR distributions}

Figure 1 shows the plots of the srnTBR values fit to BGMMs with increasing number of components. We find a significant difference in the modeling of the distribution of the srnTBR values from the 1-mode BGMM to the 2-mode BGMM ( $p$-value $=0.005$ ). There is no significant difference in the modeling of the distribution between the 2-mode and 3-mode BGMM ( $p$-value $=0.850$ ). The threshold that most accurately distinguishes between the two clusters in the 2-mode BGMM is calculated to be 1.925 for the srnTBR values. Note that for non-normalized values, this threshold should be squared, giving a threshold of 3.706 . 
Using Ward's clustering method to cluster the data into two groups, a threshold of 2.008 is defined to separate the srnTBR values, as shown in Figure 2. Note that when using this threshold on non-normalized TBR values, this threshold should be squared to get a value of 4.032 .

The false positive rate (FPR), true positive rate (TPR), and accuracy of several thresholds are shown in the ROC curve presented in Figure 3. Note that this ROC curve is calculated from the 2-mode BGMM that models the srnTBR values, however the results are shown with respect to non-normalized TBRs. The ROC curve shows that the BGMM threshold and the 35\% threshold are the most accurate thresholds in differentiating between the two clusters, with an accuracy of $79.2 \%$. The other thresholds have lower accuracy rates, however this is compensated by a lower FPR.

Table 4. Each threshold shown in the ROC curve and the percent of patients that would be classified as having an elevated TBR if this threshold were used.

\begin{tabular}{|c|l|l|}
\hline Threshold & \multicolumn{1}{|c|}{ Threshold Value } & \multicolumn{1}{|c|}{$\begin{array}{c}\text { \% observations } \\
\text { (patients) above } \\
\text { threshold }\end{array}$} \\
\hline BGMM threshold & 3.706 & $36 \%$ \\
\hline $35 \%$ threshold & 3.752 & $35 \%$ \\
\hline Ward threshold & 4.032 & $29 \%$ \\
\hline $10 \%$ FPR threshold & 4.129 & $28 \%$ \\
\hline 4.5 threshold & 4.5 & $24 \%$ \\
\hline 5 threshold & 5 & $19 \%$ \\
\hline
\end{tabular}

Table 4 shows the percent of ADHD patients that are grouped in the elevated TBR group from each threshold compared in the ROC curve. 


\section{Discussion}

\subsection{Results and thresholds}

The BGMM clustering analysis shows that the 2-mode model fits the distribution of srnTBR significantly better than the 1 -mode model ( $p$-value $=0.005)$. The 3 -mode BGMM has a larger log likelihood value than the 2-mode BGMM, however it does not model the data significantly better $(p$-value $=0.850)$. In accordance with Occam's razor, we chose the simplest model that shows a significant improvement in modelling of the data to be the best-fitting model. Note that adding components to the model will almost always increase the log likelihood value, however we want to check the significance of this increase to avoid overfitting. We conclude that the 2-mode BGMM best represents the distribution of TBR values, suggesting that there exists two distinct clusters of ADHD patients, separated by their TBR values. This suggests that there is a cluster of ADHD patients with an elevated TBR.

The confirmation of an elevated TBR cluster provides additional evidence to support the idea that ADHD is a heterogeneous disorder. This is consistent with previous literature that shows heterogeneity in ADHD (Arns et al., 2008, 2012, Barry et al., 2003; Clarke et al., 2011; Liechti et al., 2013; Loo et al., 2013, 2018).

Since our results provide evidence that ADHD is a heterogeneous disorder, all ADHD patients won't necessarily respond to the same treatment; personalized treatment may be more effective in optimizing treatment results for each patient. The existence of two distinct clusters within ADHD patients supports idea that NFT should be broken up into two subgroups: TBR down and SMR upward. This two-protocol model for NFT is a way of personalizing treatment to optimize its efficacy. This personalized method was suggested in particular by The Neurofeedback Collaborative Group (Collaborative Neurofeedback Group et al., 2013) and Arns et al. (Arns et al., 2012), and was used in a recent clinical trial to test the effectiveness of personalized NFT (NCT02778360, Mensia Technologies, France, ClinicalTrials.gov). 
Mensia Technologies' clinical trial used a TBR-threshold of 4.5 to assign ADHD subjects to an SMR increasing or TBR decreasing protocols. As shown on the ROC curve in Figure 3, a threshold of 4.5 would result in an accuracy of $75.7 \%$. The FPR of the 4.5 threshold would be around half that of the BGMM threshold, however the TPR would also be substantially decreased. Assuming the goal of the threshold is to maximize accuracy, the BGMM threshold would be the best threshold to use, with an accuracy of $79.2 \%$.

The threshold calculated from the 2-mode BGMM results in $36 \%$ of ADHD patients getting grouped in the elevated TBR cluster. This percent is consistent with many previous studies that all conclude that the percent of ADHD patients with elevated TBRs is in the range of $25-40 \%$ (Arns et al., 2012). Specifically, Clarke finds that $35 \%$ of ADHD patients have an elevated TBR, and the EEG profile of this population indicates similar deficit in processing (Clarke et al., 2011). Zhang recently uses this finding to set a threshold at $35 \%$ that separates an elevated TBR group from a control TBR group. This method of setting a threshold at $35 \%$ is supported by the results in this study, since the threshold calculated using the BGMM also defines close to the top $35 \%$ of the ADHD TBR values as being the cluster of elevated TBRs.

Previous research that studies EEG-defined subgroups of children with ADHD uses Ward's method of clustering (Clarke et al., 2011). Ward's method of clustering is used here for comparison to the results obtained from BGMM clustering. The threshold obtained from Ward's clustering is close to the threshold obtained from the BGMM threshold (4 vs. 3.7), providing additional evidence that there exists two clusters that are accurately separated by the 3.7 BGMM threshold.

When choosing a threshold for protocol assignment for NFT, the most accurate threshold is not necessarily the best choice. If we wanted to optimize accuracy, we would choose the BGMM threshold of 3.7 to be the threshold that assigns patients to the TBR protocol or the SMR protocol. If we assume, however, that the TBR decreasing protocol is more effective for patients in the elevated TBR group, while the SMR increasing protocol is equally effective for all ADHD patients, we would want to put more weight on minimizing the FPR than maximizing the accuracy of the threshold. If we set a reasonable FPR of $10 \%$, we get a threshold of 4.1 , which yields a TPR of $67 \%$ and an accuracy of 
$78 \%$. We recommend a threshold of 4.1 as the protocol assignment threshold for NFT because it has a good balance of having a low FPR and a high accuracy and TPR.

\subsection{Assumptions and limitations}

One of the assumptions made to use BGMMs to model the distribution of TBRs is that the TBRs distribute normally. A BGMM fit to a non-normal distribution would falsely identify several clusters to model the data. BGMMs assume gaussianity; it would take at least 2 Gaussian distributions to fit a skewed distribution. To make sure the normality assumption is valid, we examined the distribution of TBRs of healthy patients (from the CMI HBN database) with different normalization techniques: no normalization, lognormalization, and square-root-normalization.

After comparing different normalization techniques, we found that square-root normalization is the best technique to use to normalize the distribution of TBRs. The distributions of non-normalized, log-normalized, and srnTBR of healthy patients were examined with histograms and probability plots comparing the distributions to normal distributions (Figure 4). A Shapiro-Wilk test (Shapiro et al., 1965) was used to get a quantitative measure of normality for the control TBRs. With an alpha value of .01 , the srnTBR distribution was the only distribution for which there was not enough evidence to reject the null hypothesis of normality ( $p$-value $=0.46$ ). Thus, we assume the srnTBR is normally distributed. For future studies, if normalization is required, square-root normalization should be used to normalize TBRs.

TBR calculations in literature are astonishingly inconsistent, with values ranging from around 2 to 8 for control patients (Arns et al., 2012; Schutte et al., 2017). Part of the reason for this wide range of values is the inconsistent definitions of TBR. TBR can refer to either the ratio of powers $\left(\mu \mathrm{V}^{2}\right)$, power densities $\left(\mu \mathrm{V}^{2} / \mathrm{Hz}\right)$, voltage $(\mu \mathrm{V})$, or voltage densities $(\mu \mathrm{V} / \mathrm{Hz})$, all of which would result in a different value. TBR can be also computed from the temporal domain (like this study) or from the spectral domain (using the Fourier transform), both approaches being linked by the Parseval's theorem. The frequency bands used to calculate the TBR are also defined in different ways. For example, the TBR can come from standard frequency bands or personalized frequency bands that depend 
on the patient's IAPF. To be able to compare TBR values obtained from separate studies, a standard TBR definition needs to be set. The following definition of TBR is recommended: the ratio of power in the theta band to the power in the beta band, with the theta band defined as $[\mathrm{iAPF}-5 \mathrm{~Hz}, \mathrm{iAPF}-1 \mathrm{~Hz}]$ and the beta band defined as $[\mathrm{iAPF}+3 \mathrm{~Hz}$, $\mathrm{iAPF}+12 \mathrm{~Hz}]$.

It is important to note that this study compares results from TBRs calculated with personalized frequency bands on eyes-open to results from previous literature that use TBRs calculated with standardized frequency bands on eyes-closed (Zhang et al., 2017, Clarke et al., 2011). These TBR values are slightly different, however we assume the trends in distribution and clustering observed should be consistent.

A possible source of error in this study could be that no blink correction was used to take out blink artifacts in the EEG recordings. These blink artifacts could affect the power calculations and, in effect, the TBR calculations. However, Figure 5 shows almost no difference between TBR distributions without and with eye blink correction using blind source separation (Barthélemy et al., 2017). So, it is unlikely that these blink artifacts significantly affected the results in this study.

Another possible source of inaccuracy could be the age of the subjects included in this study. Indeed, previous literature has shown that TBR values depend on the children's age (Liechti et al., 2013; Snyder et al. 2015; Perone et al., 2018). Besides, a Mann-Whitney U-Test performed on our data shows that there is a significant difference in age between the high and low TBR clusters ( $p$-value $=2.30 \mathrm{e}-15, \mathrm{U}=22104$, respective median age \pm the median absolute deviation of each cluster: $8.0 \pm 1.57$ and $10.1 \pm 2.43$ ). So, in order to take into account that relation, the analysis was performed a second time on a restricted age range: only children between 7 and 13 years old were kept. The results obtained on this population lead to the same conclusion as previously: two clusters have been significantly identified by the BGMM ( $p$-value between 1 and 2 modes BGMM = $2.10^{-4}$ ) with a threshold of 3.84 , which is very close to the one obtained on all the data. As variations are expected to increase as a function of age, it would have been interesting to run the analysis on subgroups of youngest (5 - 6 years old) and oldest (18 - 21 years old), but because of a too limited sample size, this analysis could not be performed. The 
importance of age will be further investigated in a follow-up study in which TBR values will be age corrected in a way that will ensure the correction is not confounded by clinical symptoms. Indeed, this follow-up study will also be the occasion to investigate if there is a significant difference in the clinical symptoms between the two TBR clusters, as shown in existing literature (Buyck et al., 2014; Heinrich et al., 2014; Farnia et al., 2018).

\section{Conclusion}

The aim of this study was to investigate the existence of clusters of ADHD patients based on their TBRs computed on eyes open. The results suggest that there exist two distinct clusters of ADHD patients, characterized by an elevated TBR (with a srnTBR mean of 2.19 and a standard deviation of 0.38 ) or a normal TBR (with a srnTBR mean of 1.63 and a standard deviation of 0.31 ). Although the distributions of these two clusters overlap, the most accurate threshold to separate the two clusters is 3.7. The confirmation of these clusters has important implications for the use of NFT to treat ADHD. The existence of the clusters suggests that a two-protocol model for NFT treatment may be more effective than a single NFT protocol in optimizing treatment for each individual patient. A threshold of 4.1 is recommended as the protocol assignment threshold for such a NFT model because it has a good balance of having a low FPR as well as a high accuracy and TPR.

\section{Acknowledgments}

We would like to precise that this work was conducted thanks to the EU H2O2O NEWROFEED grant number 684809 and the Association Nationale Recherche et Technologie (ANRT) who partially funds the PhD thesis of the first author.

We would like to thank Marco Congedo, $\mathrm{PhD}$ who provided useful advice to conduct this work. We also want to thank Michael Milham, MD, PhD, for sharing the Child Mind Institute EEG databases, and the Scientific Committee of Newrofeed (S.B., H.B.F., D.P.O., D.B., T.R.) for authorizing the use of NEWROFEED EEG data. 


\section{Conflict of Interest}

A.B., Q.B., D.O. and L.M. work for Mensia Technologies. This work was part of S.C.'s internship at Mensia Technologies. During the last two years S.B. received speaker or invitation for congress from Mensia, AVAD and SOS Oxygene. In the last two years, H.B.F has received lecture fees from AB-Biotics, Rovi, Praxis, and Shire. He has been paid by Praxis for the elaboration of an article. He is the recipient of a FIPSE Grant. During the last two years, D.P.O. was principal investigator of the NEWROFEED trial without personal financement and received speaker or invitations for congresses from Shire, Medice, HAC Pharma and Otsuka.

The remaining authors declare that the research was conducted in the absence of any commercial or financial relationships that could be construed as a potential conflict of interest.

\section{Disclosure statement}

All authors approved the final article.

\section{Authors contribution}

D.O., Q.B. and L.M. contributed to the conception and design of the project, A.B. and S.C. contributed to its execution. The analysis and interpretation of the results were mainly conducted by S.C. and A.B., and validated by D.O., Q.B. and L.M.. S.B., H.B., D.B., D.P.O. and T.R. provided design input and data and commented on the article draft. L.M. and D.O. supervised the project.

\section{References}

Alexander LM, Escalera J, Ai L, Andreotti C, Febre K, Mangone A, Vega-Potler N, Langer 
$\mathrm{N}$, Alexander A, Kovacs M, Litke S. An open resource for transdiagnostic research in pediatric mental health and learning disorders. Sci Data. 2017;4:170181. https://doi.org/10.1038/sdata.2017.181.

Alexander LM. Functional Connectomes Project International Neuroimaging Data-

Sharing Initiative [dataset 1]. Available from: http://dx.doi.org/10.15387/CMI_HBN (2017b).

American Psychiatric Association. Diagnostic and statistical manual of mental disorders (DSM-5®). American Psychiatric Pub; 2013.

Antshel KM, Hargrave TM, Simonescu M, Kaul P, Hendricks K, Faraone SV. Advances in understanding and treating ADHD. BMC Med. 2011;9:72. https://doi.org/10.1186/1741-7015-9-72.

Arnold LE. Double-Blind 2-Site Randomized Clinical Trial of Neurofeedback for ADHD. Ohio State University. ClinicalTrials.gov registration number: NCT02251743. 2016. Arns M, Gunkelman J, Breteler M, Spronk D. EEG phenotypes predict treatment outcome to stimulants in children with ADHD. J Integr Neurosci. 2008;7:421-38. https://doi.org/10.1142/s0219635208001897.

Arns M, Drinkenburg W, Kenemans JL. The effects of QEEG-informed neurofeedback in ADHD: an open-label pilot study. Appl Psychophysiol Biofeedback. 2012;37:171-80. https://doi.org/10.1007/s10484-012-9191-4.

Arns M, Conners CK, Kraemer HC. A decade of EEG theta/beta ratio research in ADHD: a meta-analysis. J Atten Disord. 2013;17:374-83. https://doi.org/10.1177/1087054712460087.

Arns M, Heinrich H, Strehl U. Evaluation of neurofeedback in ADHD: the long and 
winding road. Biol Psychol. 2014;95:108-15.

https://doi.org/10.1016/j.biopsycho.2013.11.013.

Attias H. A variational bayesian framework for graphical models. Adv Neural Inf Process Syst. 2000;209-215.

Barry RJ, Clarke AR, Johnstone SJ. A review of electrophysiology in attentiondeficit/hyperactivity disorder: I. Qualitative and quantitative electroencephalography. Clin Neurophysiol. 2003;114:171-83. https://doi.org/10.1016/s1388-2457(02)00362-0.

Barthélemy Q, Mayaud L, Ojeda D, Congedo, M. The Riemannian Potato Field: a tool for online Signal Quality Index of EEG. IEEE Trans Neural Syst Rehabil Eng. 2019:27:244-255. https://doi.org/10.1109/TNSRE.2019.2893113.

Barthélemy Q, Mayaud L, Renard Y, Kim D, Kang SW, Gunkelman J, Congedo M. Online denoising of eye-blinks in electroencephalography. Neurophysiol Clin. 2017;47:371-91. https://doi.org/10.1016/j.neucli.2017.10.059.

Bazanova O, Aftanas L. Using individual EEG peculiarities increase neurofeedback efficiency. Ann General Psychiatr. 2006;5:S98. https://doi.org/10.1186/1744-859X5-S1-S98.

Bazanova OM, Aftanas LI. Individual measures of electroencephalogram alpha activity and non-verbal creativity. Neurosci Behav Physiol. 2008;38:227-35. https://doi.org/10.1007/s11055-008-0034-y.

Blei DM, Jordan MI. Variational inference for Dirichlet process mixtures. Bayesian Anal. $2006 ; 1: 121-43$.

Buyck I, Wiersema JR. Resting electroencephalogram in attention deficit hyperactivity 
disorder: developmental course and diagnostic value. Psychiatry Res. 2014;216:391-7. 10.1016/j.psychres.2013.12.055.

Chang Z, Quinn PD, Hur K, Gibbons RD, Sjölander A, Larsson H, D'Onofrio BM. Association between medication use for attention-deficit/hyperactivity disorder and risk of motor vehicle crashes. JAMA psychiatry. 2017;74:597-603. https://doi.org/10.1001/jamapsychiatry.2017.0659.

Clarke AR, Barry RJ, McCarthy R, Selikowitz M. EEG-defined subtypes of children with attention-deficit/hyperactivity disorder. Clin Neurophysiol. 2001;112:2098-105. https://doi.org/10.1016/S1388-2457(01)00668-X.

Clarke AR, Barry RJ, Dupuy FE, Heckel LD, McCarthy R, Selikowitz M, Johnstone SJ. Behavioural differences between EEG-defined subgroups of children with attention-deficit/hyperactivity disorder. Clin Neurophysiol. 2011;122:1333-41. https://doi.org/10.1016/j.clinph.2010.12.038.

Centers for Disease Control and Prevention, Centers for Disease Control and Prevention, 31 May 2017, https://www.cdc.gov/ncbddd/adhd/diagnosis.html. Last access: 2018 Oct.

Corder GW, Foreman DI. Nonparametric statistics: A step-by-step approach. John Wiley \& Sons; 2014.

Cortese S, Ferrin M, Brandeis D, Holtmann M, Aggensteiner P, Daley D, Santosh P, Simonoff E, Stevenson J, Stringaris A, Sonuga-Barke EJ. Neurofeedback for attention-deficit/hyperactivity disorder: meta-analysis of clinical and neuropsychological outcomes from randomized controlled trials. J Am Acad Child Psy. 2016;55:444-55. https://doi.org/10.1016/j.jaac.2016.03.007. 
Daley D, Van der Oord S, Ferrin M, Danckaerts M, Doepfner M, Cortese S, SonugaBarke EJ, European ADHD Guidelines Group. Behavioral interventions in attention-deficit/hyperactivity disorder: a meta-analysis of randomized controlled trials across multiple outcome domains. J Am Acad Child Adolesc Psychiatry. 2014;53:835-47. https://doi.org/10.1016/j.jaac.2014.05.013.

Escolano C, Navarro-Gil M, Garcia-Campayo J, Congedo M, Minguez J. The effects of individual upper alpha neurofeedback in ADHD: an open-label pilot study. Applied psychophysiology and biofeedback. 2014;39:193-202.

https://doi.org/10.1007/s10484-014-9257-6.

Epstein JN, Loren RE. Changes in the definition of ADHD in DSM-5: subtle but important. Neuropsychiatry. 2013;3:455. https://doi.org/10.2217/npy.13.59.

Evans SW, Schultz BK, DeMars CE, Davis H. Effectiveness of the Challenging Horizons after-school program for young adolescents with ADHD. Behav Ther. 2011;42:462-74. https://doi.org/10.1016/j.beth.2010.11.008.

Farnia S, Alipour A, Alaghbandrad J, beygum Shafaat A, Fattahi S, Ghahremanloo M. Investigating the Relationship between Theta/Beta Ratio and Intensity of Disease in Children with ADHD. Frontiers in Biomedical Technologies. 2018;5:18-29.

Heinrich H, Busch K, Studer P, Erbe K, Moll GH, Kratz O. EEG spectral analysis of attention in ADHD: implications for neurofeedback training? Front Hum Neurosci. 2014;8:611. https://doi.org/10.3389/fnhum.2014.00611.

Hernandez LD, Rieger K, Koenig T. Low motivational incongruence predicts successful EEG resting-state neurofeedback performance in healthy adults. Neuroscience. 2016. https://doi.org/10.1016/j.neuroscience.2016.12.005 
Jangmo A, Stålhandske A, Chang Z, Chen Q, Almqvist C, Feldman I, Bulik CM, Lichtenstein P, D’Onofrio B, Kuja-Halkola R, Larsson H. AttentionDeficit/Hyperactivity Disorder, School Performance, and Effect of Medication. J Am Acad Child Adolesc Psychiatry. 2019;58:423:32. https://doi.org/10.1016/j.jaac.2018.11.014.

James G, Witten D, Hastie T, Tibshirani R. An introduction to statistical learning. New York: springer; 2013.

Kaiser DA. Rethinking standard bands. J Neurother. 2001;5:87-96. https://doi.org/10.1300/J184v05n01_08.

Kaufman J, Birmaher B, Brent D, Rao UM, Flynn C, Moreci P, Williamson D, Ryan N. Schedule for affective disorders and schizophrenia for school-age children-present and lifetime version (K-SADS-PL): initial reliability and validity data. J Am Acad Child Psy. 1997;36:980-8. https://doi.org/10.1097/00004583-199707000-00021. The Collaborative Neurofeedback Group, Arnold LE, Arns M, Conners K, deBeus R, Hirshberg L, Kerson C, Kraemer H, Lofthouse N, Lubar J, McBurnett K, Monastra V. A proposed multisite double-blind randomized clinical trial of neurofeedback for ADHD: need, rationale, and strategy. J Atten Disord. 2013;17:420-36. https://doi.org/10.1177/1087054713482580.

Klimesch W. EEG alpha and theta oscillations reflect cognitive and memory performance: a review and analysis. Brain Res Rev. 1999;29:169-95. https://doi.org/10.1016/s0165-0173(98)00056-3.

Langer N, Ho EJ, Alexander LM, Xu HY, Jozanovic RK, Henin S, Petroni A, Cohen S, Marcelle ET, Parra LC, Milham MP. A resource for assessing information 
processing in the developing brain using EEG and eye tracking. Sci Data. 2017;4:170040. https://doi.org/10.1038/sdata.2017.40.

Langer N. 1000 Functional Connectomes Project International Neuroimaging DataSharing Initiative (FCP/INDI) [dataset 2]. Available from: http://dx.doi.org/10.15387/FCP_INDI.MIPDB.eeg (2017b).

Lichtenstein P, Halldner L, Zetterqvist J, Sjölander A, Serlachius E, Fazel S, Långström $\mathrm{N}$, Larsson $\mathrm{H}$. Medication for attention deficit-hyperactivity disorder and criminality. N Engl J Med. 2012;367:2006-14.

Liechti MD, Valko L, Müller UC, Döhnert M, Drechsler R, Steinhausen HC, Brandeis D. Diagnostic value of resting electroencephalogram in attention-deficit/hyperactivity disorder across the lifespan. Brain Topogr. 2013;26:135-51. https://doi.org/10.1007/s10548-012-0258-6.

Loo SK, Cho A, Hale TS, McGough J, McCracken J, Smalley SL. Characterization of the theta to beta ratio in ADHD: identifying potential sources of heterogeneity. $J$ Atten Disord. 2013;17:384-92. https://doi.org/10.1177/1087054712468050.

Loo SK, McGough JJ, McCracken JT, Smalley SL. Parsing heterogeneity in attentiondeficit hyperactivity disorder using EEG- based subgroups. J Child Psychol Psyc. 2018;59:223-31. https://doi.org/0.1111/jcpp.12814.

Marzbani H, Marateb HR, Mansourian M. Neurofeedback: a comprehensive review on system design, methodology and clinical applications. Basic Clin Neurosci. 2016;7:143. https://doi.org/10.15412/J.BCN.03070208.

Mayer K, Blume F, Wyckoff SN, Brokmeier LL, Strehl U. Neurofeedback of slow cortical potentials as a treatment for adults with Attention Deficit-/Hyperactivity Disorder. 
Clin Neurophysiol. 2016;127:1374-86.

https://doi.org/10.1016/j.clinph.2015.11.013.

Mensia Technologies SA, Paris, France. Identifier NCT02778360, Effectiveness of a Personalized Neurofeedback Training Device (ADHD@Home) as Compared With Methylphenidate in the Treatment of Children and Adolescents With AttentionDeficit/Hyperactivity Disorder: A Multicentre Randomized Clinical Study. ClinicalTrials.gov registration number: NCT02778360. 2017.

Micoulaud-Franchi JA, Geoffroy PA, Fond G, Lopez R, Bioulac S, Philip P. EEG neurofeedback treatments in children with ADHD: an updated meta-analysis of randomized controlled trials. Front Hum Neurosci. 2014;8:906.

https://doi.org/10.3389/fnhum.2014.00906.

Murphy AC, Bassett DS. A network neuroscience of neurofeedback for clinical translation. Curr Opin Biomed Eng. 2017;1:63-70. https://doi.org/10.1016/j.cobme.2017.03.003.

National Institute of Mental Health, U.S. Department of Health and Human Services, https://www.nimh.nih.gov/health/topics/attention-deficit-hyperactivity-disorderadhd/index.shtml. Last access: 2018 Oct.

Neba Health (2015). Neba. https://shop.nebahealth.com/products/neba-fda-clearedbrainwave-adhd-assessment-aid. Last access: 2018 Oct.

Nigg JT, Holton K. Restriction and elimination diets in ADHD treatment. Child Adol Psych Cl. 2014;23:937-53. https://doi.org/10.1016/j.chc.2014.05.010.

Pappas D. ADHD Rating Scale-IV: Checklists, norms, and clinical interpretation. J Psychoeduc Assesst. 2006;24:172-8. https://doi.org/10.1177/0734282905285792 
Pedregosa F, Varoquaux G, Gramfort A, Michel V, Thirion B, Grisel O, Blondel M,

Prettenhofer P, Weiss R, Dubourg V, Vanderplas J. Scikit-learn: Machine learning in Python. J Mach Learn Res. 2011;12:2825-30.

Perone S, Palanisamy J, Carlson SM. Age-related change in brain rhythms from early to middle childhood: Links to executive function. Dev Sci. 2018;21:e12691. https://doi.org/10.1111/desc.12691.

Polanczyk G, De Lima MS, Horta BL, Biederman J, Rohde LA. The worldwide prevalence of ADHD: a systematic review and metaregression analysis. Am J Psychiat. 2007;164:942-8. https://doi.org/10.1176/ajp.2007.164.6.942.

Saad JF, Kohn MR, Clarke S, Lagopoulos J, Hermens DF. Is the theta/beta EEG marker for ADHD inherently flawed?. J Atten Disord. 2015. https://doi.org/10.1177/1087054715578270.

Schutte I, Kenemans JL, Schutter DJ. Resting-state theta/beta EEG ratio is associated with reward-and punishment-related reversal learning. Cog Affect Behav Neurosci. 2017;17:754-63. https://doi.org/10.3758/s13415-017-0510-3.

Shapiro SS, Wilk MB. An analysis of variance test for normality (complete samples). Biometrika. 1965;52:591-611. https://doi.org/10.2307/2333709.

Sonuga- Barke EJ, Thompson M, Daley D, Laver- Bradbury C. Parent training for Attention Deficit/Hyperactivity Disorder: is it as effective when delivered as routine rather than as specialist care?. Brit J Clin Psychol. 2004 ;43:449-57. https://doi.org/10.1348/0144665042388973.

Sonuga-Barke EJ, Brandeis D, Cortese S, Daley D, Ferrin M, Holtmann M, Stevenson J, Danckaerts M, Van der Oord S, Döpfner M, Dittmann RW. Nonpharmacological 
interventions for ADHD: systematic review and meta-analyses of randomized controlled trials of dietary and psychological treatments. Am J Psychiat. 2013;170:275-89. https://doi.org/10.1176/appi.ajp.2012.12070991.

De Sousa A, Kalra G. Drug therapy of attention deficit hyperactivity disorder: Current trends. Mens Sana Monogr. 2012;10:45. https://doi.org/10.4103/0973-1229.87261.

Storebø OJ, Krogh HB, Ramstad E, Moreira-Maia CR, Holmskov M, Skoog M, Nilausen TD, Magnusson FL, Zwi M, Gillies D, Rosendal S. Methylphenidate for attentiondeficit/hyperactivity disorder in children and adolescents: Cochrane systematic review with meta-analyses and trial sequential analyses of randomised clinical trials. BMJ. 2015;351:h5203. https://doi.org/10.1136/bmj.h5203.

Snyder SM, Rugino TA, Hornig M, Stein MA. Integration of an EEG biomarker with a clinician's ADHD evaluation. Brain Behav. 2015;5:e00330. https://doi.org/10.1002/brb3.330.

Swanson JM, Arnold LE, Molina BSG, Sibley MH, Hechtman LT, Hinshaw SP, Abikoff H B, Stehli A, Owens EB, Mitchell, JT, Nichols Q, Howard A, Greenhill LL, Hoza B, Newcorn JH, Jensen PS, Vitiello B, Wigal T, Epstein JN, Tamm L, Lakes KD, Waxmonsky J, Lerner M, Etcovitch J, Murray DW, Muenke M, Acosta MT, ArcosBurgos M, Pelham WE, Kraemer HC, \& the MTA Cooperative Group. Young adult outcomes in the follow-up of the multimodal treatment study of attentiondeficit/hyperactivity disorder: symptom persistence, source discrepancy, and height suppression. J Child Psychol Psychiatry. 2017;58:663-78. https://doi.org/10.1111/jcpp.12684.

U.S. Food and Drug Administration. De novo classification request for Neuropsychiatric 
EEG-Based Assessment Aid for ADHD (NEBA) system. 2013. Retrieved from http://www. accessdata.fda.gov/cdrh_docs/reviews/K112711.pdf.

Van Doren J, Arns M, Heinrich H, Vollebregt MA, StrehI U, Loo SK. Sustained effects of neurofeedback in ADHD: a systematic review and meta-analysis. Eur Child Adolesc Psychiatry. 2018;1:1-3.

Vollebregt MA, van Dongen-Boomsma M, Slaats-Willemse D, Buitelaar JK, Oostenveld R. How the individual alpha peak frequency helps unravel the neurophysiologic underpinnings of behavioral functioning in children with attentiondeficit/hyperactivity disorder. Clin EEG Neurosci. 2015;46:285-91. https://doi.org/10.1177/1550059414537257.

Ward Jr JH. Hierarchical grouping to optimize an objective function. J. Am. Stat. Assoc. 1963;58:236-44.

https://doi.org/10.1080/01621459.1963.10500845

Welch P. The use of fast Fourier transform for the estimation of power spectra: a method based on time averaging over short, modified periodograms. IEEE Transactions on audio and electroacoustics. 1967;15:70-3. https://doi:10.1109/TAU.1967.1161901

Wechsler D. Manual for the Wechsler abbreviated intelligence scale (WASI). San Antonio, TX: The Psychological Corporation. 1999.

Zhang DW, Roodenrys S, Li H, Barry RJ, Clarke AR, Wu Z, Zhao Q, Song Y, Liu L, Qian Q, Wang Y. Atypical interference control in children with AD/HD with elevated theta/beta ratio. Biol Psychol 2017;128:82-8. https://doi.org/10.1016/j.biopsycho.2017.07.009. 


\section{Figures}
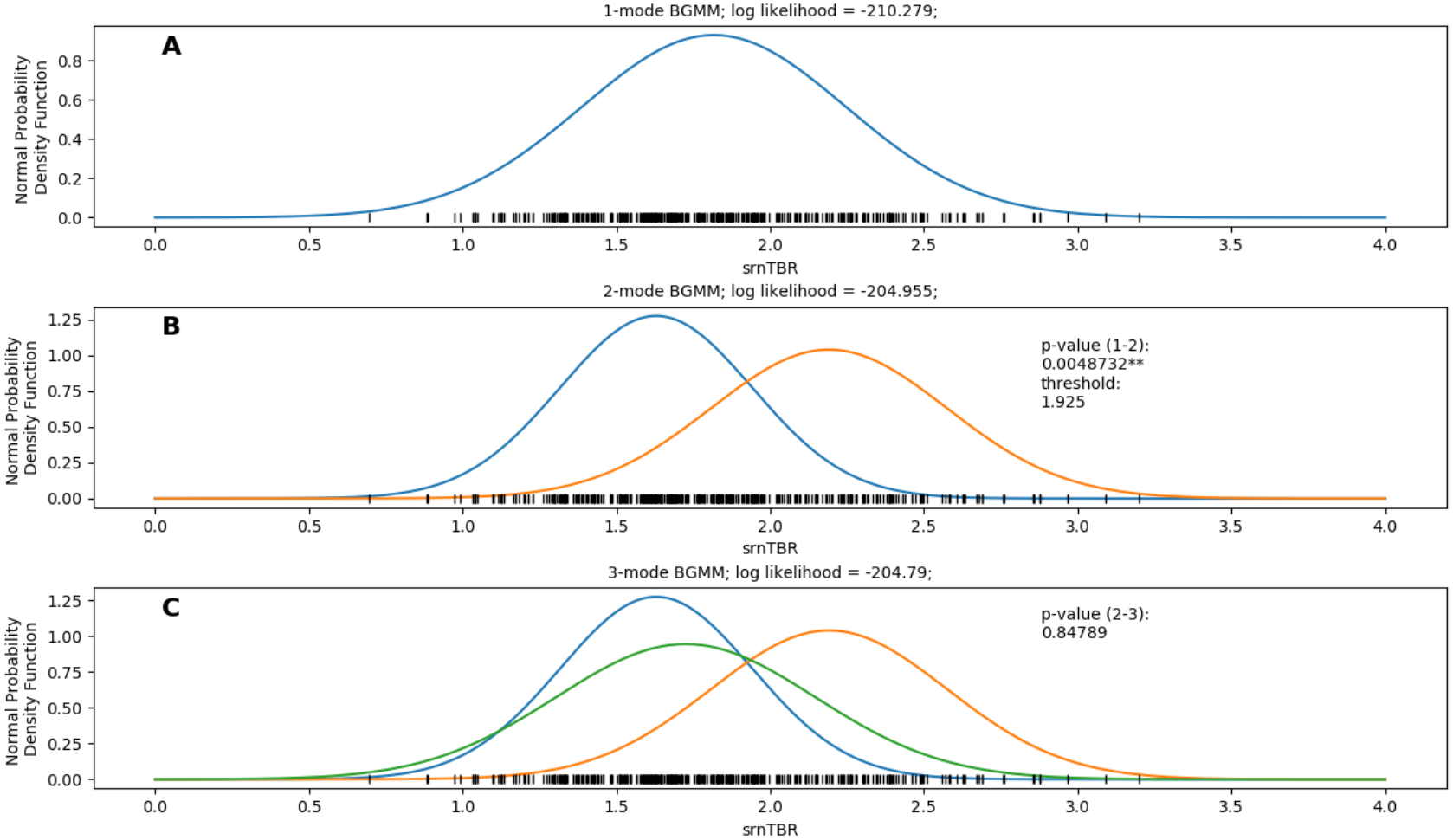

Figure 1. BGMM - srnTBR. Plots of srnTBR distribution (rug plot) with a A) 1-mode, B) 2-mode, and C) 3mode BGMM superimposed. P-value (1-2) = p-value from deviance test comparing the 1-mode and 2mode BGMMs; $p$-value (2-3) = p-value from deviance test comparing the 2-mode and 3-mode BGMMs; ** p-value $<0.01$. 


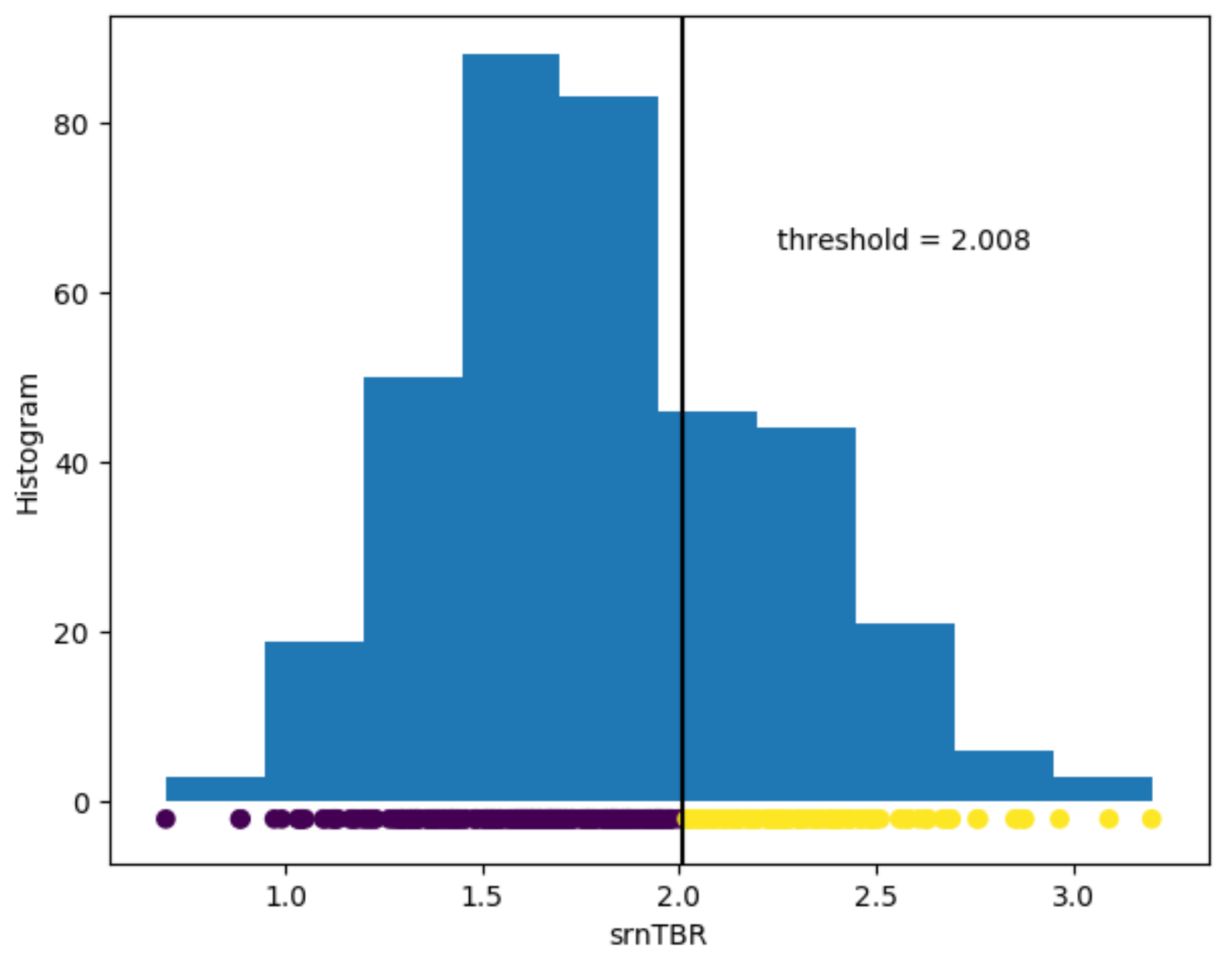

Figure 2. Ward method clustering of ADHD srnTBR values. Plot showing the distribution of srnTBR values with a histogram and each value plotted below, color-coordinated to distinguish between clusters identified by Ward's method of clustering. srnTBR value is shown on the x-axis; subject count is shown on the $y$-axis; the threshold is represented by the vertical black line. 


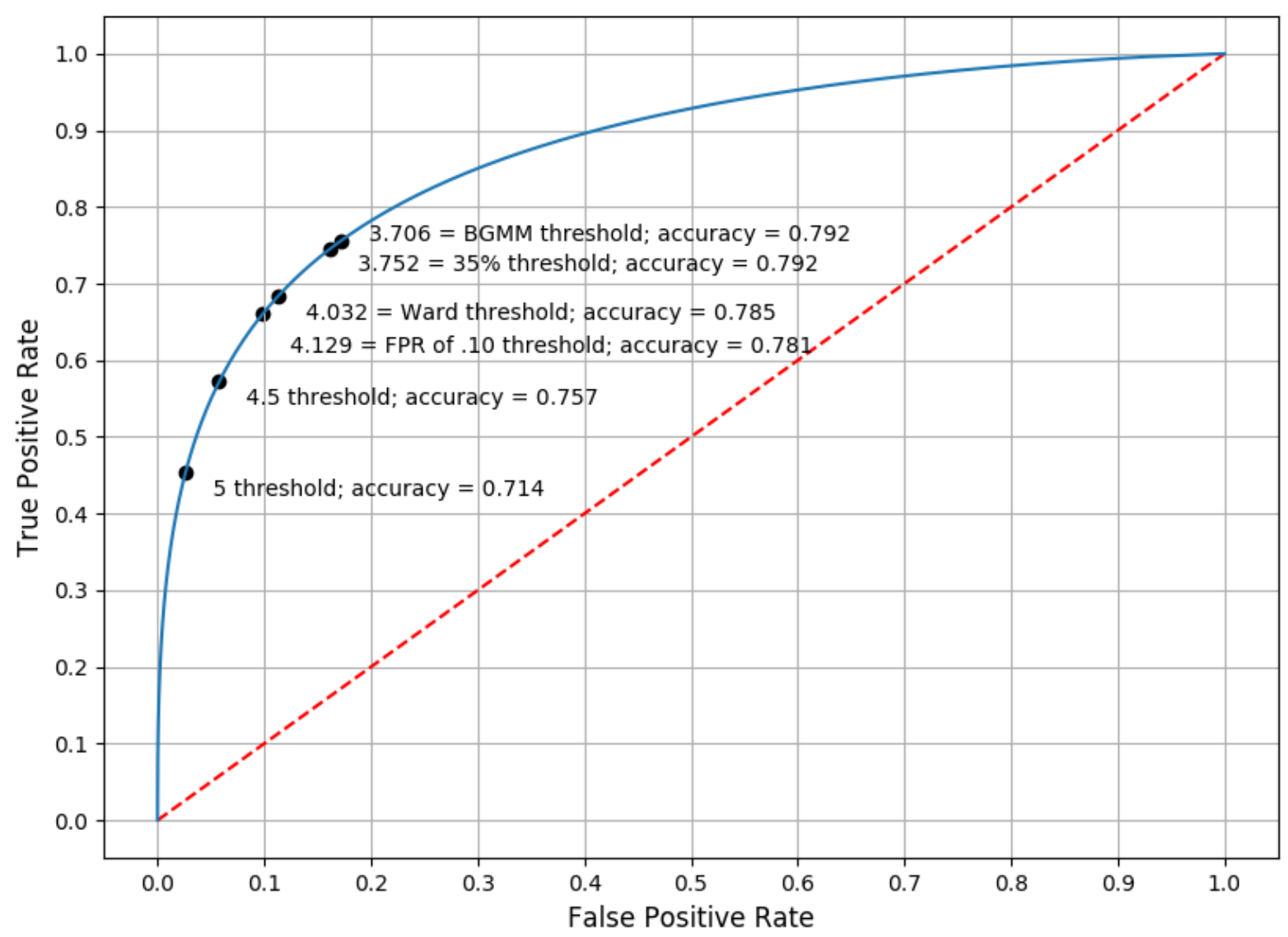

Figure 3. ROC Curve; $A \cup C=0.872$. Plot of $\mathrm{ROC}$ curve with various possible thresholds shown with their respective accuracies. $A \cup C=$ area under the curve. 

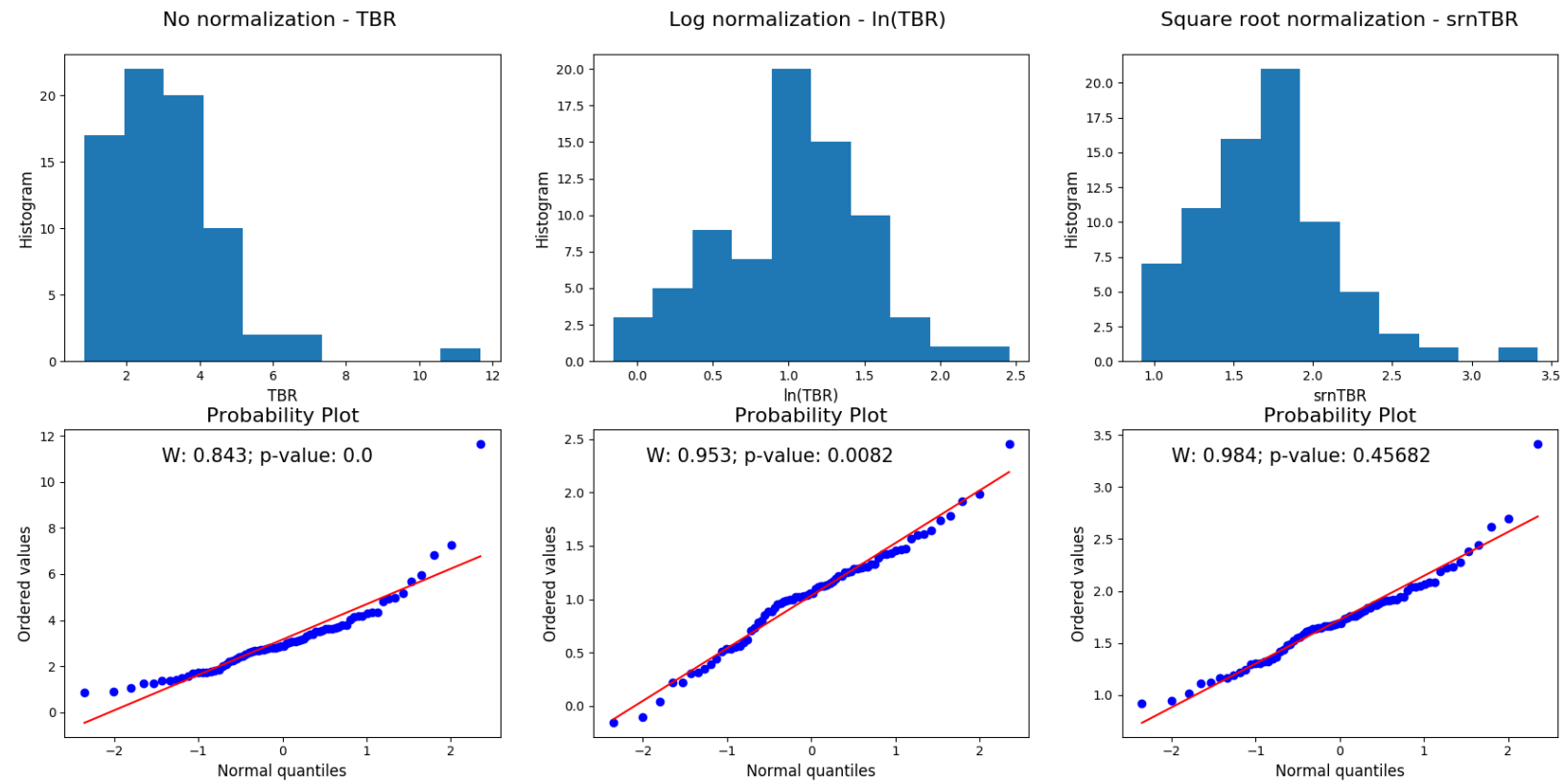

Figure 4. Comparison of normalizations in healthy TBRs. Plots of histograms of TBRs with no normalization, log normalization, and square-root normalization, shown in the first row. The second row shows the probability plot comparing the above. Shapiro-Wilk test statistic W and p-value are displayed on each probability plot. 


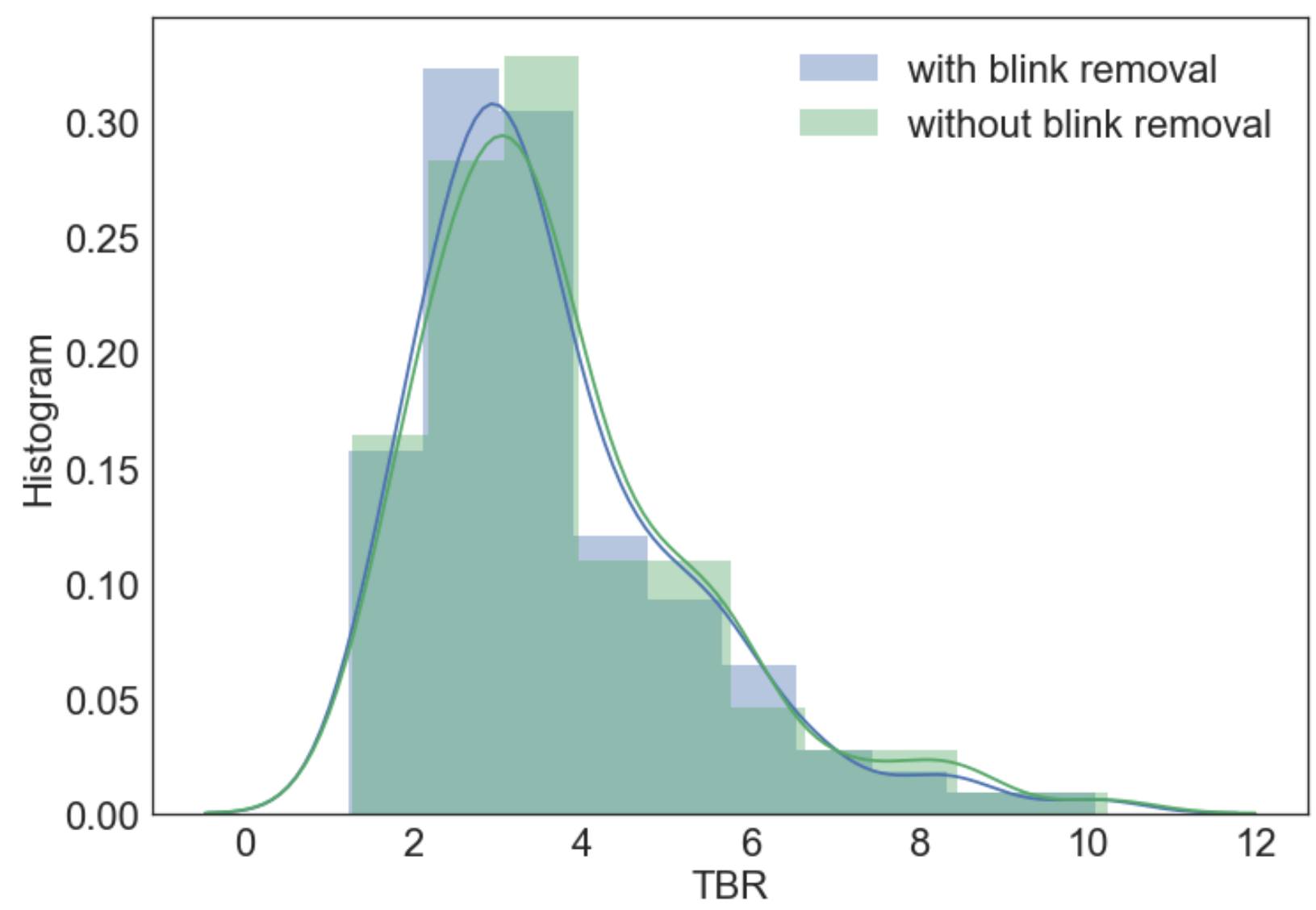

Figure 5. Comparison of TBR distributions in ADHD patients computed with and without blink removal. 